\title{
Citología y Análisis Morfométrico de las Células del Epitelio Vaginal Durante el Ciclo Estral en Ovejas de Pelo (Ovis aries)
}

\author{
Citology and Morphometric Analysis of Epithelial Cells from the \\ Vagina of Hair Sheep Ewes (Ovis aries) During their Estrous Cycle
}

Neftali Clemente Ovando; Agustín Orihuela Trujillo; Fernando Iván Flores Pérez \& Virginio Aguirre Flores

CLEMENTE, O. N.; ORIHUELA, T. A.; FLORES, P. F. I. \& AGUIRRE, F. V. Citología y análisis morfométrico de las células del epitelio vaginal durante el ciclo estral en ovejas de pelo (Ovis aries). Int. J. Morphol., 31(3):888-893, 2013.

RESUMEN: La citología vaginal puede usarse para caracterizar estados del ciclo reproductivo de la hembra. Sin embargo, no ha sido validada en ovinos de pelo, y ofrece resultados inexactos debido a interpretaciones subjetivas. El objetivo del presente trabajo es tipificar las características morfológicas y morfométricas de las células epiteliales de la vagina durante el ciclo estral en ovinos de pelo. Cinco borregas Santa Cruz se sincronizaron con esponjas intravaginales. Desde el día del 1er celo posterior al retiro de la esponja y hasta el siguiente celo, se obtuvieron muestras diarias del epitelio vaginal de cada oveja, y se cuantificó el número de células parabasales, intermedias y superficiales, y se midió la longitud, perímetro y área de citoplasma y núcleo de cada una. Los resultados muestran que las células superficiales incrementan $(\mathrm{P}<0,05)$ durante el estro en comparación con el diestro. En contraste, el número de células parabasales e intermedias es mayor $(\mathrm{P}<0,05)$ durante el diestro que en el estro. Las células más grandes $(36,11 \pm 0,38 \mu \mathrm{m})$ y núcleos más pequeños $(8,46 \pm 0,12 \mu \mathrm{m})$ se encontraron durante el estro, independientemente del tipo de célula, mientras que citoplasmas más pequeños (26,72 $\pm 0,11$ $\mu \mathrm{m})$ y núcleos más grandes $(10,48 \pm 0,02 \mu \mathrm{m})$ se observaron durante el diestro. Se concluye que la morfología de las células epiteliales de la vagina de la oveja de pelo coincide con las variaciones encontradas en otras hembras, y que el tamaño de citoplasmas y núcleos presenta un patrón cíclico que puede utilizarse para determinar de manera objetiva la etapa del ciclo estral de la oveja.

PALABRAS CLAVE: Epitelio vaginal; Citología; Ciclo estral; Ovinos de pelo; Santa Cruz.

\section{INTRODUCCIÓN}

El tracto reproductor femenino se encuentra bajo la influencia de diversas hormonas sexuales que actúan sobre las células epiteliales (Meikle et al., 2001). Estas hormonas promueven eventos como la proliferación, diferenciación y muerte celular (Graham \& Clarke, 1997; O'Brien et al., 2006) que se observan mediante citología vaginal exfoliativa en diversas especies animales a lo largo del ciclo estral (Miroud \& Noakes, 1990; Bouchard et al., 1991; Ola et al., 2006).

En los trabajos de citología vaginal exfoliativa de manera general se clasifican las células epiteliales que se desprenden de la pared vaginal en: células parabasales, intermedias y superficiales. En esta clasificación se emplea fundamentalmente un criterio morfológico y de afinidad tintorial (Hayashi et al., 1988), que al ser meramente descriptivo, presenta la desventaja de tener variaciones relacionadas con el ejecutor de la técnica (Moxon et al., 2010).
Aunado a lo anterior, en ovinos existe muy poca información sobre citología vaginal exfoliativa que describa el comportamiento de las células epiteliales durante el ciclo estral completo. Además de que estos trabajos se han realizado en razas originarias de latitudes altas y climas templados exclusivamente (Sanger et al., 1958; Ghannam et al., 1972), por lo que la técnica no se ha validado en ovejas de pelo de origen tropical.

Por otra parte, hoy en día, las herramientas computacionales ofrecen la posibilidad de aplicar la morfometría como una herramienta útil en la descripción de los tipos celulares existentes en la pared vaginal de las hembras durante el ciclo estral (Dutta et al., 2011) lo que permitirá disminuir el error de interpretación de las muestras citológicas. Sin embargo, la morfometría no se ha realizado, así como tampoco se ha establecido si existe una relación entre la variación en los tamaños celulares 
asociada a los diferentes tipos de células presentes en las diferentes etapas del ciclo estral. Por lo que en el presente estudio se propone además de usar el criterio de clasificación celular, efectuar un análisis morfométrico que permita describir de manera cuantitativa los cambios morfológicos que tienen las células epiteliales de la pared vaginal de ovinos de pelo durante el ciclo estral.

\section{MATERIAL Y MÉTODO}

Se utilizaron cinco hembras multíparas de la raza Santa Cruz, con condición corporal, peso y edad

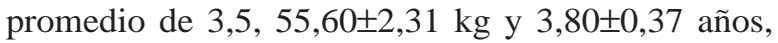
respectivamente. Las ovejas permanecieron estabuladas en un corral de $8 \mathrm{~m}^{2}$, y se alimentaron con $2 \mathrm{~kg}$ de pasto Taiwán (Pennisetum purpureum) picado y $0,40 \mathrm{~kg}$ de alimento comercial con $14 \%$ de proteína por animal por día. El agua y sales se ofrecieron a libre demanda.

Las hembras se indujeron al celo mediante la inserción de esponjas intravaginales impregnadas con $30 \mathrm{mg}$ de Acetato de Flurogestona* (*IntervetSchering-Plough Animal Health, México) durante 12 días. Veinticuatro horas después de haber retirado las esponjas se introdujo diario al corral de las hembras un macho equipado con mandil a las 08:00 y 16:00 h para detectar el celo.

Las hembras se consideraron en celo cuando aceptaban ser montadas por el macho.

Obtención de las muestras. A partir de que cada hembra se detectó en celo y hasta la aparición del segundo celo, diario a las 09:00 h se tomó una muestra individual para evaluación de la citología vaginal exfoliativa.

La muestra se obtuvo separando los labios de la vulva y se introdujo un espéculo lubricado con el fin de localizar la zona situada entre la entrada del cérvix y la vagina. Hecho esto, se colectó la muestra con un hisopo de algodón estéril, en la pared vaginal con el fin de extraer las células que se desprenden de manera natural. Se retiró el hisopo y se trazaron dos líneas paralelas sobre un portaobjetos impregnando las células en la superficie de acuerdo a la metodología realizada por Ola et al.

Preparación y evaluación de las muestras citológicas. La fijación de las células, se efectuó su- mergiendo el portaobjeto en alcohol al 95\% durante $10 \mathrm{~min}$ y secando a temperatura ambiente. Las células se tiñeron con Hematoxilina-Eosina (Raposo \& da Silva, 1999).

La observación de las células se efectuó con un microscopio óptico en cada muestra citológica y se llevó acabo con el objetivo de 40X. En cada muestra citológica se evaluaron seis campos microscópicos que correspondieron a un área de 948,7 $\mu \mathrm{m}^{2}$ cada uno, tomados de la parte del centro y extremos de las líneas paralelas de material impregnado en los portaobjetos. Los campos fueron fotografiados para garantizar que la evaluación se llevara acabo en el mismo campo visual.

Las células epiteliales encontradas en los campos fueron clasificadas de acuerdo al criterio de Grunert en parabasales, intermedias y superficiales (Schutte, 1967). Con la finalidad de reducir la variación en la interpretación de las citologías, las evaluaciones se llevaron a cabo por dos operarios, registrando los promedios de ambas determinaciones.

La medición y conteo celular se realizó tomando una fotografía de cada uno de los seis campos microscópicos con una cámara digital* (*Motic Imagen Plus Versión 2.0 ML. Motic China Group CO., China) montada en el ocular del microscopio, posteriormente, las fotografías se analizaron por medio del programa de análisis métricos* (*Motic Images Plus Versión 2.0 ML. Motic China Group CO., China), en donde se midió la longitud (diámetro mayor), perímetro y área del citoplasma y núcleo de cada una de las células presentes en los campos de visión (Fig. 1).

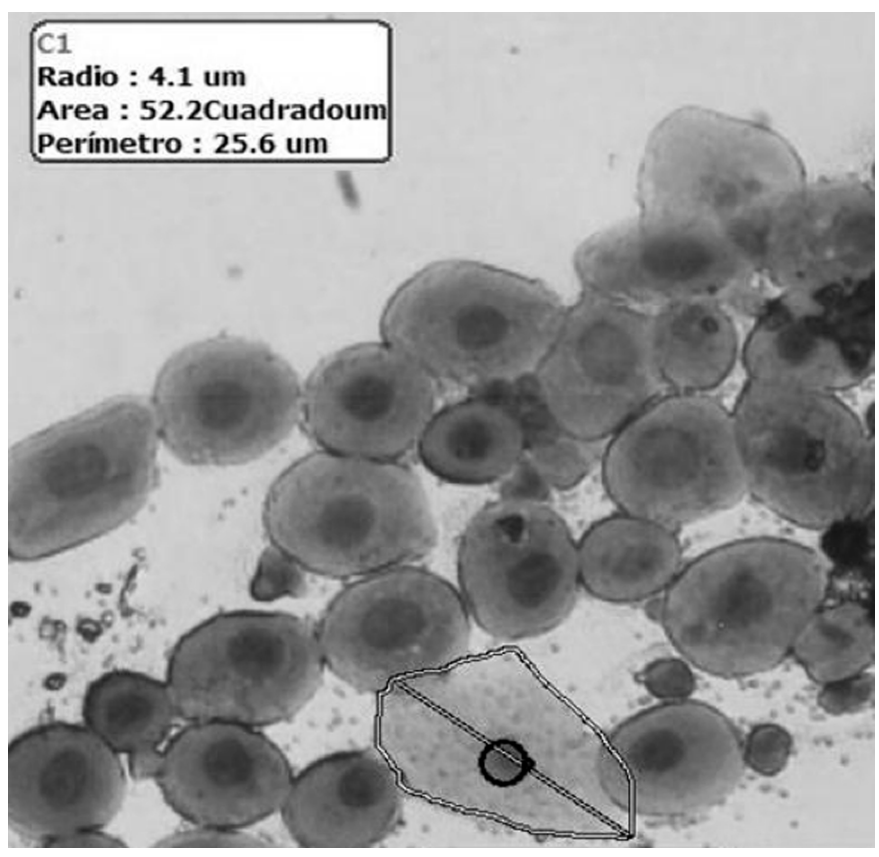

Fig. 1. Fotografía apreciando la medición (longitud, perímetro y área del citoplasma y núcleo) de las células epiteliales de la pared vaginal. 
La medición celular se relacionó con las etapas del ciclo estral, donde se consideró al estro como el momento en que la oveja se dejó montar $\pm 12 \mathrm{~h}$. Metaestro como la fase siguiente, con una duración de 48 h. Diestro, se consideró a partir del final del metaestro, y hasta $48 \mathrm{~h}$ antes del siguiente estro, y el proestro, comprendió $48 \mathrm{~h}$ antes del siguiente estro.

Análisis de datos. Los datos de la evaluación morfológica, conteo y medición celular se agruparon en estro, metaestro, diestro y proestro, se analizaron mediante un análisis de varianza de una vía y posteriormente mediante una prueba de comparación múltiple de Bonferroni. Además, se realizó una prueba de correlación entre los valores obtenidos de las variables longitud, área y perímetro del citoplasma y núcleo con el programa estadístico GraphPad Prism 4.

\section{RESULTADOS}

El mayor número de células superficiales se observó durante la etapa de estro $(35,47 \pm 5,86$ células) y metaestro $(28,90 \pm 7,01$ células) en comparación con el diestro $(6,04 \pm$ 1,43 células). Las células intermedias disminuyeron durante el estro (23,07 $\pm 5,16$ células) en comparación con el metaestro (50,50 $\pm 5,34$ células) y el diestro $(58,76 \pm 2,85$ células). Así mismo, las células parabasales se encontraron en menor cantidad durante el estro $(5,73 \pm 2,45$ células $)$ y el proestro $(9,70$ $\pm 3,00$ células), que durante el metaestro (32,30 $\pm 3,96$ células) y el diestro (46,27 $\pm 2,67$ células) (Fig. 2).
Las características morfométricas de las células epiteliales variaron a lo largo del ciclo estral (Tabla I), los citoplasmas de mayor longitud, perímetro y área se observaron durante el estro. Los núcleos más pequeños, se encontraron durante el estro, en comparación con las demás etapas del ciclo estral. El tamaño de los núcleos fue aumentando hasta registrar su mayor tamaño durante el diestro, para después disminuir en el proestro. Se observó una correlación entre núcleo y citoplasma de $\mathrm{r}=-0,74,-0,73$ y -0,64 en las variables de longitud, perímetro y área, respectivamente.

La menor cantidad de células por campo se observó durante el estro $(64,26 \pm 11,75$ células) y el proestro $(59,00$ $\pm 7,89$ células), en comparación con el metaestro (111,7 \pm 8,18 células) y el diestro ( $111,09 \pm 4,90$ células). La duración promedio del ciclo estral fue de 17,20 \pm 0,37 días.

\section{DISCUSIÓN}

Los patrones celulares observados en la citología vaginal exfoliativa coinciden con los referidos en ovejas de lana (Sanger et al.), cabras (Pérez-Martinez et al.,1999; Ola et al.) y perras (Bouchard et al.)

La explicación fisiológica de los patrones celulares observados en el presente estudio se debe a la acción de estradiol y progesterona sobre el epitelio vaginal ya que los primeros causan proliferación, estratificación y cornificación del epitelio vaginal (Buchanan et al., 1998). Sin embargo,

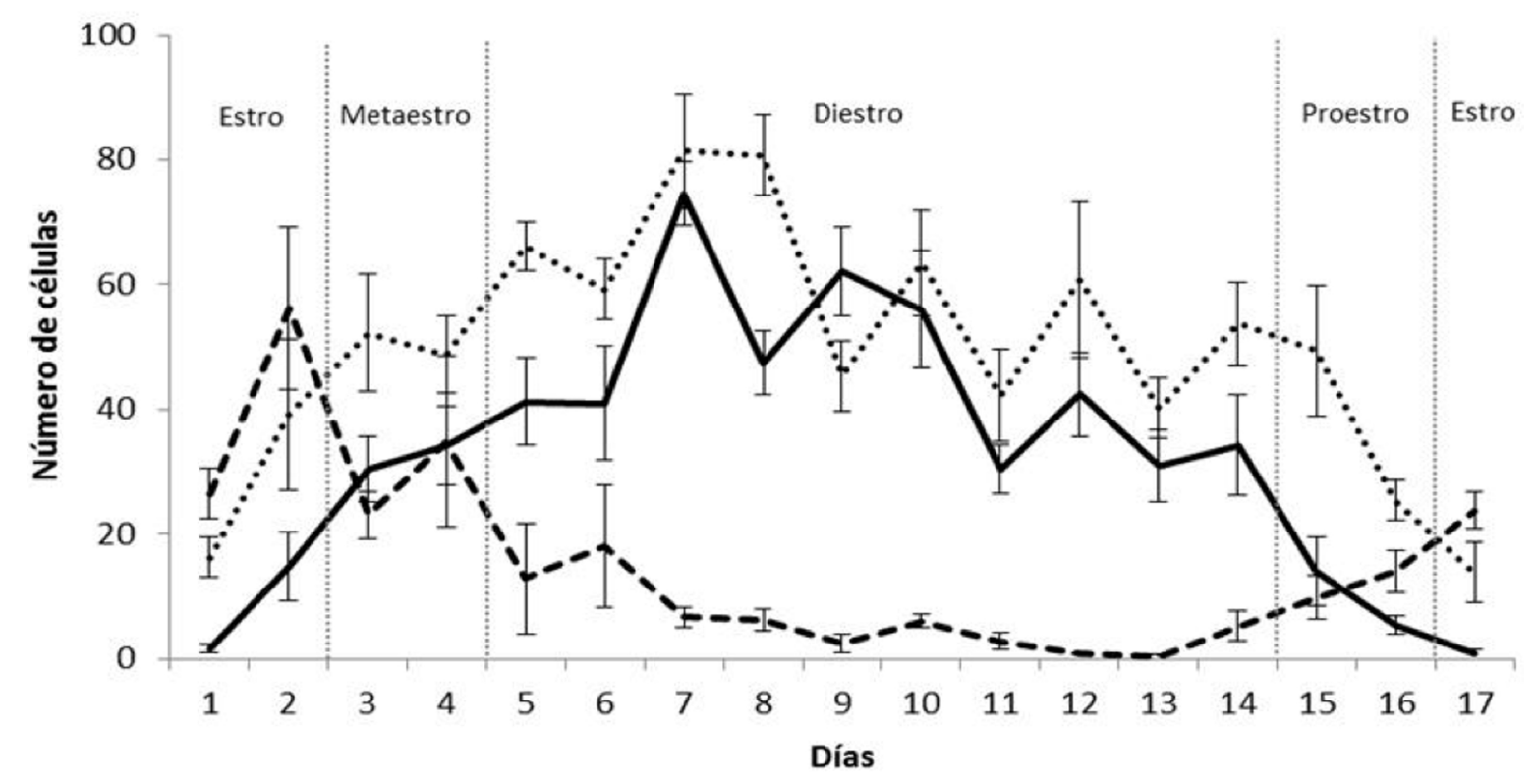

Fig. 2. Número de células (promedio \pm ee) parabasales (-), intermedias (…) y superficiales (---) observadas durante las etapas del ciclo estral de ovejas Santa Cruz. 
CLEMENTE, O. N.; ORIHUELA, T. A.; FLORES, P. F. I. \& AGUIRRE, F. V. Citología y análisis morfométrico de las células del epitelio vaginal durante el ciclo estral en ovejas de pelo (Ovis aries). Int. J. Morphol., 31(3):888-893, 2013.

Tabla I. Valores promedio ( \pm ee) de las dimensiones (micrómetros) de las células epiteliales vaginales.

\begin{tabular}{lcccccc}
\hline & \multicolumn{3}{c}{ Citoplasma } & \multicolumn{2}{c}{ Núcleo } \\
\hline \multirow{2}{*}{ Estro } & Longitud & Perímetro & Área & Longitud & Perímetro & Área \\
\cline { 2 - 7 } Mestestro & $36,11 \pm 0,38 \mathrm{a}$ & $110,70 \pm 1,09 \mathrm{a}$ & $727,80 \pm 13,79^{\mathrm{a}}$ & $8,46 \pm 0,12^{\mathrm{c}}$ & $26,57 \pm 0,41 \mathrm{c}$ & $63,28 \pm 1,20^{\mathrm{c}}$ \\
Diestro & $30,53 \pm 0,38 \mathrm{~b}$ & $94,38 \pm 1,11 \mathrm{~b}$ & $569,30 \pm 12,11^{\mathrm{b}}$ & $9,08 \pm 0,06^{\mathrm{b}}$ & $28,66 \pm 0,22^{\mathrm{b}}$ & $66,85 \pm 0,96^{\mathrm{b}, \mathrm{c}}$ \\
Proestro & $26,72 \pm 0,11 \mathrm{c}$ & $83,82 \pm 0,36 \mathrm{c}$ & $479,30 \pm 3,59 \mathrm{c}$ & $10,48 \pm 0,02^{\mathrm{a}}$ & $32,96 \pm 0,10 \mathrm{a}$ & $86,85 \pm 0,45^{\mathrm{a}}$ \\
& $29,57 \pm 0,40 \mathrm{~b}$ & $92,17 \pm 1,15 \mathrm{~b}$ & $542,90 \pm 13,05^{\mathrm{b}}$ & $9,07 \pm 0,14^{\mathrm{b}}$ & $28,42 \pm 0,43^{\mathrm{b}}$ & $71,12 \pm 1,58 \mathrm{~b}$ \\
\hline
\end{tabular}

a,b,c Diferentes literales dentro de una misma columna indican diferencias significativas $(\mathrm{P}<0,05)$.

estos efectos varían dependiendo del estrato celular, por ejemplo en estratos basales causa proliferación celular, y sobre estratos intermedios y luminales provoca la queratinización y exfoliación de estas células (Gupta et al., 1989; Pessina et al., 2006).

Por otra parte, la disminución en el tamaño de las células observadas en los patrones de citología en el presente estudio, estaría dada por la progesterona que tiene efectos opuestos a los inducidos por los estrógenos (Rider, 2002) al intervenir en la disminución de la síntesis de receptores a estrógenos (Meikle et al., 2004), y controlar los efectos proliferativos del factor de crecimiento insulínico (IGF-1), a través de incrementar los niveles del factor de crecimiento insulínico ligado a proteínas tipo 1 (IGFBP-1) que inhiben al IGF-1 (Graham \& Clarke; Fowler et al., 2000).

Mills et al. (1979) al realizar citología vaginal en gatas y medir las células por medio de un micrómetro ocular durante las distintas etapas de su ciclo estral, hacen evidentes los cambios inducidos por las hormonas estradiol y progesterona, y refiere que las células predominantes durante la etapa del estro fueron las de mayor tamaño de citoplasma registrado, con un promedio de $68,5 \pm 10,5 \mu \mathrm{m}$ de longitud, además también poseían el núcleo mas pequeño con 7,9 $\pm 1,2 \mu \mathrm{m}$ de longitud. En cambio en las etapas de metaestro tardío, anestro y proestro células de tamaño medio y con núcleo grande (citoplasma 44,7士8,9 y núcleo $10,0 \pm 2,1 \mu \mathrm{m}$ de longitud) fueron las predominantes.

Asimismo, Centola et al. (1978) al realizar citología vaginal exfoliativa y observar el material colectado mediante el microscopio electrónico de barrido encontraron que células de mayor tamaño (32 $\mu \mathrm{m}$ de longitud), predominaron durante la etapa del estro en la rata.

Estos resultados son similares a los encontrados en el presente trabajo donde las células de mayor tamaño (citoplasma $36,1 \pm 0,38 \mu \mathrm{m}$ longitud) y con núcleo más pequeño $(8,46 \pm 0,12 \mu \mathrm{m}$ de longitud) se hallaron en el estro y las células con citoplasma más pequeños $(26,7 \pm 0,11 \mu \mathrm{m}$ de longitud) y núcleo más grande $(10,48 \pm 0,02 \mu \mathrm{m}$ longitud) se registraron durante el diestro.
Bott et al. (2006) observaron una disminución en la cantidad de núcleos del ectocervix al administrar 17 bestradiol a ovejas ovariectomizadas, similar a lo encontrado durante la etapa del estro de hembras intactas. En contraparte, en la fase lútea de ovejas intactas y a la aplicación de progesterona a ovejas ovariectomizadas se observó un incremento en la cantidad de núcleos. Estos resultados coinciden con el presente estudio, en donde, se encontró una menor cantidad de células durante la etapa de estro en comparación con el diestro, aunque contrarios con los de Ola et al. quienes refieren un número mayor de células durante los primeros días del ciclo estral y disminuidos durante los siguientes días. Esta discrepancia podría deberse a que durante la etapa del estro existe una secreción mucosa (Sanger et al.) que dificulta la adhesión de las células a la superficie del vidrio del portaobjetos reduciendo el número de células impregnadas en él, y por consecuencia el número de células observadas al microscopio.

El análisis morfométrico celular y específicamente del núcleo, se ha utilizado como un método de diagnóstico de tumores cancerígenos (Strefezzi et al., 2004; Dutta et al.), asimismo su medición durante el ciclo menstrual de la mujer aporta información con respecto a la fase del ciclo menstrual en la que se encuentra (Strefezzi et al.), y actualmente para realizar un análisis morfométrico celular no se requiere equipos sofisticados ni costosos, además de ser sencillos de aplicar.

Se propone que la relación entre el tamaño del núcleo y citoplasma que varía durante las distintas etapas del ciclo estral de la oveja, es un parámetro más preciso en comparación con el método de clasificación morfológica tradicional para la determinar el ciclo estral en la hembra ovina.

\section{AGRADECIMIENTOS}

Al técnico Maribel Nieto Miranda por proporcionar los colorantes utilizados en este estudio y por los comentarios realizados para la ejecución de la técnica de citología vaginal exfoliativa. 
CLEMENTE, O. N.; ORIHUELA, T. A.; FLORES, P. F. I. \& AGUIRRE, F. V. Citology and morphometric analysis of epithelial cells from the vagina of hair sheep ewes (Ovis aries) during their estrous cycle. Int. J. Morphol., 31(3):888-893, 2013.

SUMMARY: Vaginal cytology can be used to characterize stages of the reproductive cycle of the female. However, it has not been validated in hair sheep, and generates inaccurate results due to subjective interpretations. Thus, the purpose of the present work is to typify morphologic and morphometric characteristics of the epithelial cells from the vagina during the estrous cycle of hair sheep. Five Saint Croix ewes were synchronized with intravaginal sponges. Day zero of the cycle was considered the day of first estrus after sponge withdrawal. From this day until next estrus, samples from the vaginal epithelium were obtained daily from each ewe. The number of basal, intermediate and superficial cells was quantified, and length, perimeter, and area from cytoplasm and nucleus were obtained. The results show that the number of superficial cells increase $(\mathrm{P}<0.05)$ during estrus and decrease during the rest of the cycle. In contrast, the number of basal and intermediate cells showed an inverse pattern. The largest cells $(36.11 \pm 0.38 \mu \mathrm{m})$ and smallest nucleuses $(8.46 \pm 0.12$ $\mu \mathrm{m})$ were present during estrus, regardless of the cell type, while the smallest cells $(26.72 \pm 0.11 \mu \mathrm{m})$ and larger nucleuses $(10.48 \pm 0.02$ $\mu \mathrm{m})$ were observed during diestrus. It was concluded that citology of the epithelial cells from the vagina of hair ewes is in accord with changes found in other female spices, and that cytoplasm and nucleus sizes display a cyclic pattern that might be used to objectively determine the specific estrous cycle phase of the ewes.

KEY WORDS: Vaginal epithelium; Cytology; Estrous cycle; Hair sheep; Saint Croix.

\section{REFERENCIAS BIBLIOGRÁFICAS}

Bott, E. M.; Young, I. R.; Jenkin, G. \& Mclaren, W. J. Detection of morphological changes of the ovine cervix in response to sex steroids using a fluorescence confocal endomicroscope. Am. J. Obstet. Gynecol., 194(1):105-12, 2006.

Bouchard, G. F.; Solorzano, N.; Concannon, P. W.; Youngquist, R. S. \& Bierschwal, C. J. Determination of ovulation time in bitches based on teasing, vaginal cytology, and Elisa for progesterone. Theriogenology, 35(3):603-11, 1991.

Buchanan, D. L.; Kurita, T.; Taylor, J. A.; Lubahn, B. D.; Cunha, G. R. \& Cooke, P. S. Role of Stromal and Epithelial Estrogen Receptors in Vaginal Epithelial Proliferation, Stratification, and Cornification. Endocrinol., 139(10):4345-52, 1998.

Centola, G. M. Surface features of exfoliated vaginal epithelial cells during the oestrous cycle of the rat examined by scanning electron microscopy. J. Anat., 127(Pt. 3):553-61, 1978.

Dutta, S.; Basu, A. \& Mukherji, B. Nuclear morphometry of perimenopausal endometrium in Nepali population. Al Ameen J. Med. Sci., 4(3):247-53, 2011.

Fowler, D. J.; Nicolaides, K. H. \& Miell, J. P. Insuline-like growth factor binding protein-1 (IGFB-1): a multifunctional role in the human female reproductive tract. Hum. Reprod. Update, 6(5):495-504, 2000.

Ghannam, S. A.; Bosc, M. J. \& du Buisson, F. Examination of vaginal epithelium of the sheep and its use in pregnancy diagnosis. Am. J. Vet. Res., 33(6):1175-85, 1972.

Graham, J. D. \& Clarke, C. L. Physiological action of progesterone in target tissues. Endocr. Rev., 18(4):502-19, 1997.

Gupta, P. D.; Vijayasaradhi, S. \& Reddy, A. G. Keratinization of rat vaginal epithelium. III. Effect of estradiol on keratinization. Biol. Cell, 65(3):281-9, 1989.

Hayashi, K.; Hayashi, M.; Boutin, E.; Cunha, G.R.; Bernfield, M. \& Trelstad, R. L. Hormonal modification of epithelial differentiation and expression of cell surface heparan sulfate proteoglycan in the mouse vaginal epithelium. An immunohistochemical and electron microscopic study. Lab. Invest., 58(1):68-76, 1988.

Meikle, A.; Garófalo, E. G.; Rodríguez-Piñón, M.; Tasende, C. \& Sahlin, L. Regulation by gonadal steroids of estrogen and progesterone receptors along the reproductive tract in female lambs. Acta Vet. Scand., 42(1):161-9, 2001.

Meikle, A.; Tasende, C.; Sosa, C. \& Gorófalo, G. E. The role of sex steroid receptors in sheep female reproductive physiology. Reprod. Fertil. Dev., 16(4):385-94, 2004.

Mills, J. N.; Valli, V. E. \& Lumsden, J. H. Cyclical changes of vaginal cytology in the cat. Can. Vet. J., 20(4):95-101, 1979.

Miroud, K. \& Noakes, D. E. Exfoliative vaginal cytology during the oestrous cycle of the cow, after ovariectomy, and after exogenous progesterone andoestradiol-17beta. Br. Vet. J., 146(5):387-97, 1990.

Moxon, R.; Copley, D. \& England, G. C. Quality assurance of canine vaginal cytology: A preliminary study. Theriogenology, 74(3):479-85, 2010.

O’Brien, J. E.; Peterson, T. J.; Tong, M. H.; Lee, E. J.; Pfaff, L. E.; Hewitt, S. C.; et al. Estrogen-induced proliferation of uterine epithelial cells is independent of estrogen receptor a binding to classical estrogen response elements. J. Biol. Chem., 281(36):26683-92, 2006. 
CLEMENTE, O. N.; ORIHUELA, T. A.; FLORES, P. F. I. \& AGUIRRE, F. V. Citología y análisis morfométrico de las células del epitelio vaginal durante el ciclo estral en ovejas de pelo (Ovis aries). Int. J. Morphol., 31(3):888-893, 2013.

Ola, S. I.; Sanni, W. A. \& Egbunike, G. Exfoliative vaginal cytology during the oestrous cycle of West African dwarf goats. Reprod. Nutr. Dev., 46(1):87-95, 2006.

Pérez-Martínez, M.; Mendoza, M. E. \& Romano, M. C. Exfoliative vaginal cytology and plasma levels of estrone and estradiol$17 \mathrm{~b}$ in young and adult goats. Small Ruminant Res., 33(2):153$8,1999$.

Pessina, M. A.; Hoyt, R. F. Jr.; Goldstein, I. \& Traish, A. M. Differential regulation of the expression of estrogen, progesterone, and androgen receptors by sex steroid hormones in the vagina: immune-histochemical studies. J. Sex. Med., 3(5):804-14, 2006.

Raposo, R. \& da Silva, L. D. M. Comparação qualitativa de diferentes técnicas de coloração para a citologia vaginal de cabras da raça Saanen. Ciênc Anim., 9(2):81-5, 1999.

Rider, V. Progesterone and the control of uterine cell proliferation and differentiation. Front. Biosci., 7:d1545-55, 2002.

Sanger, V. L.; Engle, P. H. \& Bell, D. S. The vaginal cytology of the ewe during the estrous cycle. Am. J. Vet. Res., 19(71):283$7,1958$.

Schutte, A. P. Canine vaginal cytology. I. Technique and cytological morphology. J. Small Anim. Pract., 8(6):301-6, 1967

Strefezzi, R. F.; Xavier, J. G.; Kleeb, S. R., \& Catão-Dias, J. L. Nuclear morphometry in cytopathology: a prognostic indicator for canine cutaneous mast cell tumors. J. Vet. Diagn. Invest., 21(6):821-5, 2009.

\author{
Dirección para Correspondencia: \\ Neftali Clemente Ovando \\ Facultad de Ciencias Agropecuarias \\ Centro de Desarrollo e Investigación Agropecuaria (CEDIA) \\ Universidad Autónoma del Estado de Morelos \\ Av. Universidad 1001, Col. Chamilpa \\ CP. 62210 \\ Cuernavaca, Morelos \\ MÉXICO
}

Tel: 7351653561.

Email: nefta850@hotmail.com

Recibido : 31-01-2013

Aceptado: 01-05-2013 